\title{
The Research on Freeway Fan-in Area
}

\author{
Wenqian Jiang \\ North China Electric Power University, Baoding 071000, China \\ jwq163mail@163.com
}

Keywords: cellular automaton, simulation, changing-lane, merging

\begin{abstract}
The barrier toll is a crucial part of a freeway. The bottleneck of a freeway about safety and throughput is usually the fan-in area.In this paper, I put forward the merging model of barrier tolls (MBT) based on cellular automaton(CA) taking various factors which influence the merging of vehicles into consideration.
\end{abstract}

\section{Introduction}

A barrier toll is a collection mechanism at an entrance or exit of a freeway which connects with two provinces or two states.Typically,a barrier toll has several advantages,such as the control of traffic volume and the quality of traffic flow.However,the fan-in area after the barrier toll can be a bottleneck of the freeway.As we all know,a much larger number of the barrier tolls is provided than the number of travel lanes leaving the toll plaza.So,the fan-in area is a crucial factor that influences the throughput.It will be significant that if we can make a optimum proposal about the design of the fan-in area.In this paper, I make a deeply research on the fan-in area.Taking accident prevention,throughput and cost into account, I aim to decide the optimal shape,size and merging pattern of the fan-in area.

\section{Assumptions and Justifications}

To simplify the problem, I make some assumptions.

- The time that Highway tollbooth operator serves motorists is within a certain range. I also regard this time as a stochastic figure.Due to a man is subjective,Highway tollbooth operator can not do the same action in the same time and the speed level of payment action is different with each motorist.

- All motorists tend to change lane as soon as possible while meeting conditions(If motorists need to change lane).Nearly every motorist wants drive in the middle of the freeway.

- The speed of leaving the toll barrier is the equivalent of each. when motorists pay for tolls,they pull up.After exiting the toll barriers,motorists start the vehicles.At this moment,the speed is negligible compared with that running on road.

\section{Build the Model}

I can regard the performance of the vehicle in fan-in area as going straightly and changing lanes.Leaving barrier polls that directly facing lanes of freeway,vehicles go straightly and enter correspondent lanes.Then,other vehicles have to change lanes so as to enter formal lanes.If there is more than one lane needing to change,motorists must change line one by one according to the rule of traffic. I build my model with a cellular-automaton-based approach.

\subsection{Influencing Factors}

For vehicles which need change lines,they enter formal lanes step by step.motorists not only need to consider the speed,but also the gap between itself and the latter vehicle of the neighbor lane.If motorists decelerate,vehicles face the possibility of collision with the latter vehicle of the neighboring lane.If motorists accelerate, vehicles face the possibility of collision with the former vehicle. 


\subsection{The MBT Model Based on Cellular Automaton}

Cellular Automaton(CA) modeling is extended to study the heterogeneous traffic observed in developing countries.In heterogeneous traffic,the physical and mechanical characteristics of different vehicles vary widely which in turn leads to complex traffic.This nature of the heterogeneous traffic is modeled with the help of CA model.[1]Cellular automaton is mathematical model in which time,space and other variables are all discrete.

In fan-in area,because of the influence of vehicles in neighboring lane and the shape of the lane,the rules of going straightly and changing lanes vary according to different condition.We can refer to the $\mathrm{Na}$ Sch Model.[2]Cells,like the squares of a checkerboard in the model are divided into three modes containing idle,occupied and impenetrable.Idle mode represent there is no vehicle.Occupied mode represent there is a vehicle.Impenetrable mode represent that vehicles can not get through.

For Model Based on Cellular Automaton, I make some explanations:

- Each cell is a $3.65 \mathrm{~m}^{*} 6 \mathrm{~m}$ area in reality.According to the width of lane and the length of vehicle in reality, I can surely make it.

- I operate procedure 2000 times.I can obtain a fairly correct answer.

- Vehicles are in the middle of each lane.

- I suppose that the performance of changing lane occur in a moment.

- I suppose the speeds of the vehicles are positive integer.

- Unit time is one second.

\subsubsection{The criteria of going straightly}

1) Update the site of the former vehicle.

$$
\left\{\begin{array}{cc}
x(i, j+v(i, j))=1 & x(i, j)=0 \\
x(i, m p-1)=1 & m p \leq x(i, j)+v(i, j)<\text { road_length } \\
\text { disappear } & x(i, j)+v(i, j)>\text { road_length }
\end{array}\right.
$$

2)Update the site of the latter vehicle.

$$
\left\{\begin{array}{cc}
x(i, j+v(i, j))=1 & j+v(i, j)<x(i, j))^{\prime} \\
x(i, j-1)=1 & j+v(i, j) \geq x(i, j)
\end{array}\right.
$$

3)Vary the speed of the former vehicle.

$$
v(i, j)=\left\{\begin{array}{lr}
v(i, j)+1 & p \\
v(i, j) & 1-p
\end{array}\right.
$$

4)Vary the speed of the latter vehicle.

$$
v(i, j)=\left\{\begin{array}{cc}
v(i, j)^{\prime} & x(i, j)=x(i, j)^{\prime}-1 \\
v(i, j)+1 & p \\
v(i, j) & 1-p
\end{array}\right.
$$

5)Record the track of vehicle.For example If a vehicle go straightly,the site of it is $x^{t}\left(i, j_{1}\right)$ at $t$ moment and the site of it is $x^{t+1}\left(i, j_{2}\right)$ at $t+1$ moment. So the track of the vehicle is from $j_{1}$ to $j_{2}$. I define a matrix A to record the track.As shown in the following Figure1 .

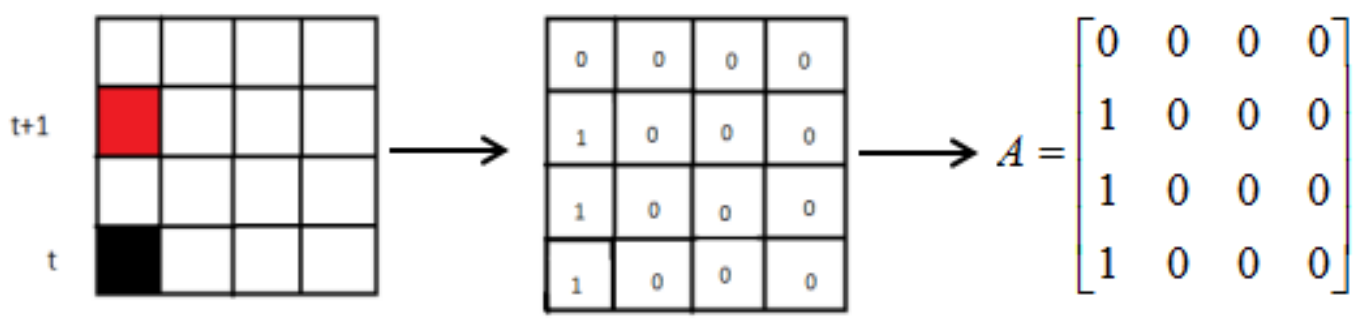

Figure 1:The track record of vehicles going straightly 


\subsubsection{The criteria of changing lanes}

1) Judge the condition of changing lanes and update the site matrix.If $A(i+1, j)=0 \& A(i+1, j+1)=0$, then $x(i+1, j+1)=1$.

2) Keep speed.

3) Record the track of vehicle.For example

If a vehicle is changing lane,the site of it is $x^{t}\left(i, j_{1}\right)$ while the speed of it is $v^{t}\left(i, j_{1}\right)$ at $t$ moment and the site of it is $x^{t+1}\left(i, j_{2}\right)$ while the speed of it is $v^{t+1}\left(i, j_{2}\right)$ at $t+1$ moment.So, I define a matrix $\mathbf{A}$ to record the track in one second.As shown in the following Figure 2 .

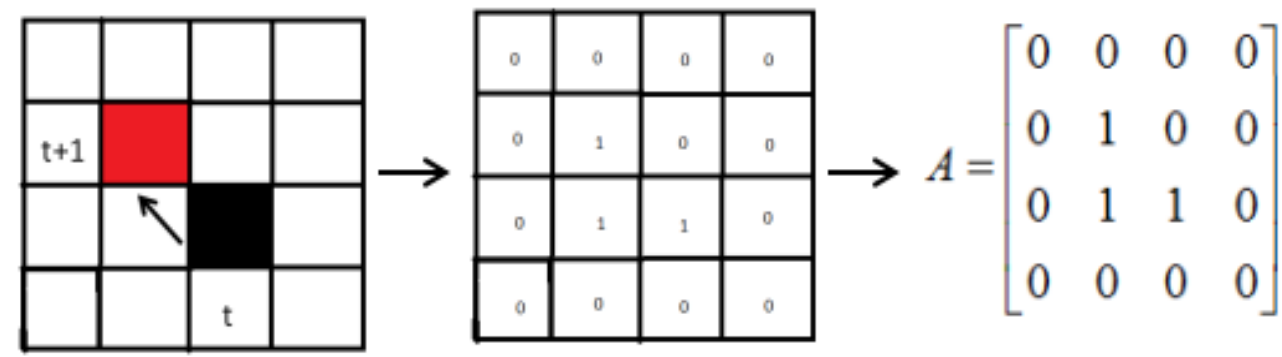

Figure 2:The track record of vehicles changing lanes

\subsubsection{Release Criteria}

Each motorist choose a barrier toll among the amount of barrier tolls randomly.The time motorists spend paying toll is in a certain range.The time appearing a new vehicle at the exit in the certain range is stochastic.

The explanation of the result of simulation at one moment.

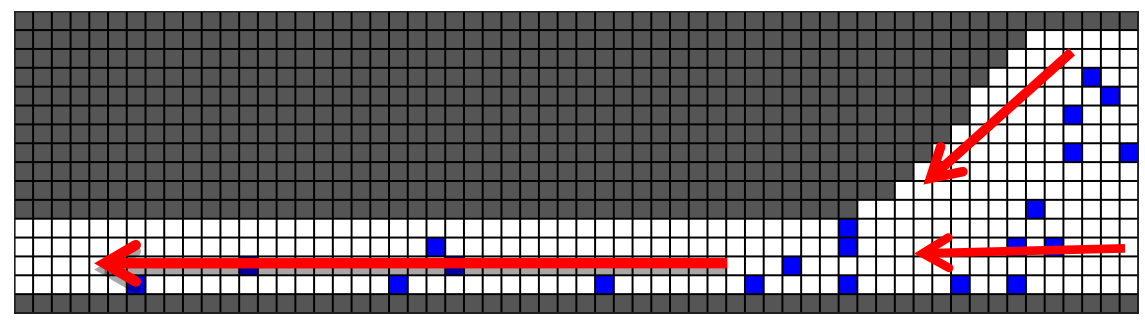

Figure 3:Explanation of simulation

\section{Conclusions}

At this section, I try my best to research the four-lane freeway in a direction.However,I can not find a function relationship between the size,shape,merging pattern and the number of barrier tolls, the number of lanes. Therefore,I obtain the solution through constantly simulation and iterative operation.I present the steps of solution with four-lane freeway in a direction.

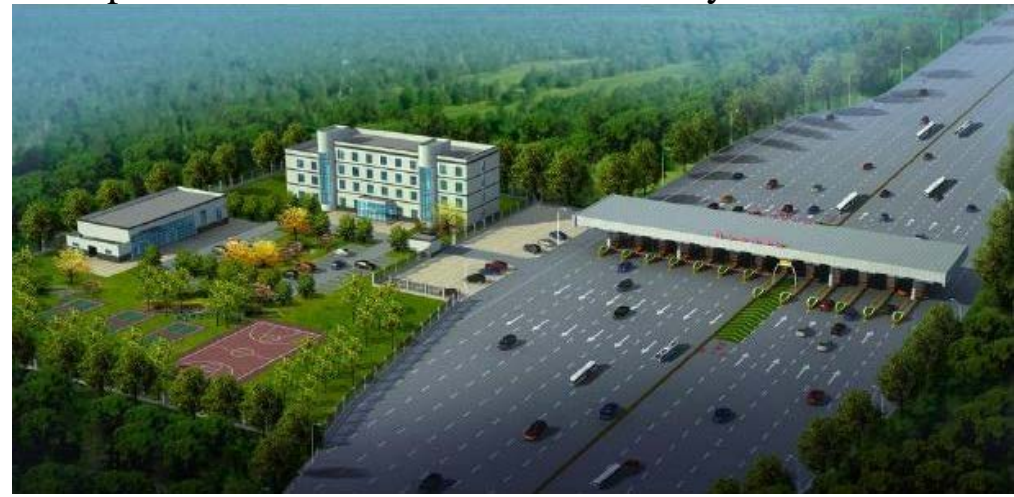

Figure 4:A real toll plaza

From:http://pic116.nipic.com/file/20161124/11088342_150652650926_2.jpg 
If the number of lanes is given, my model can derive several number of barrier tolls from the process of simulating the traffic flow on fan-in area.The solution given by model indicates that eleven-barrier-toll is most suitable for a four-lane freeway with 90000 vehicles traffic flow one day.According to the simulation of lane-changing, I can obtain the final shape like purple curve in Figure 5.
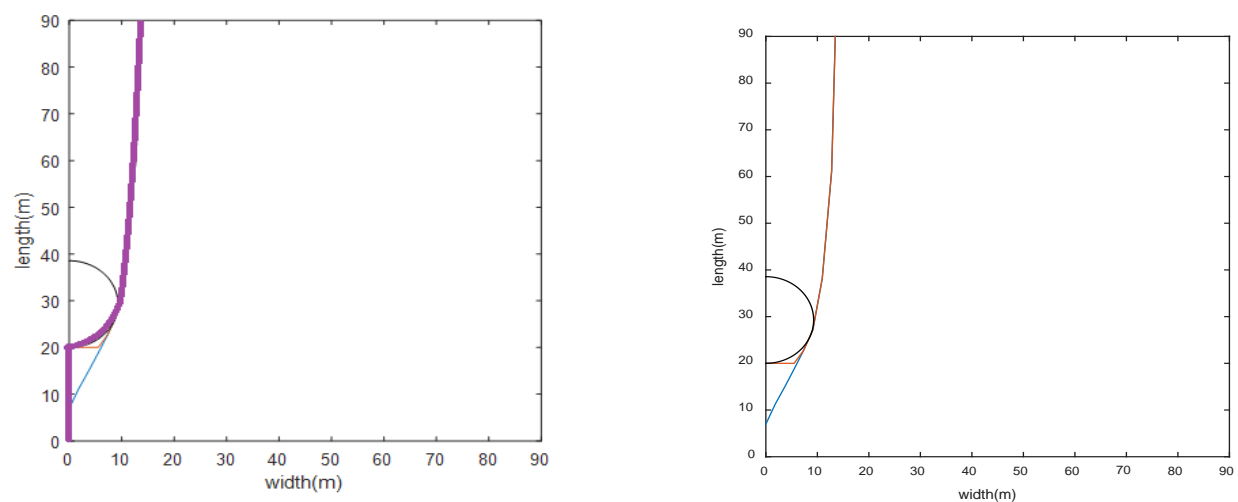

Figure 5:The final simulation design of fan-in area(four-lane and eleven-barrier-toll)

\section{Analysis of Conclusion}

\subsection{Performance in Light And Heavy Traffic}

I study the fan-in area of freeways with four-lane and eleven-barrier-toll.We give definition of light traffic and heavy traffic.The value of throughput on the behalf of the degree of congestion.The larger throughput is,the more crowded.Oppositely,the smaller throughput is,the more unhindered[3].In the model,we obtain different throughput by changing time that all barrier tolls need to pay tolls.If I decrease the time that motorists pass the barrier tolls,throughput increases and the traffic is heavier.Oppositely,if I increase the time that motorists pass the barrier tolls,throughput decreases and the traffic is lighter.

\subsection{The Performance with Different Proportions of Barrier Tolls}

Similarly,I can consider the influence on model of different proportions of conventional tollbooths,exact-change tollbooths and electronic toll collection booths.In section 6.1, what I change is the time that all barrier tolls need to pay tolls.In this section,I change the time of partial barrier tolls need to pay tolls.In this way,I can represent exact-change tollbooths and electronic toll collection booths by different number of partial barrier tolls. That is to say, the difference of conventional tollbooths,exact-change tollbooths and electronic toll collection booths is the time that all barrier tolls need to check tolls. The time of conventional tollbooths is the longest and the time of electronic toll collection booths is the shortest.

\section{References}

[1]Mallikarjuna,C\&Rao,KR Cellular Automata Model for Heterogeneous Traffic.JOURNAL OF ADVANCED TRANSPORTATION,2009:321-345.

http://apps.webofknowledge.com/Cited Full Record.do?product=WOS\&col Name=WOS\&SI D=4DUXu6Ua X2JXKh3h Y25\&search_mode=Cited Full Record\&isickref=WOS:0002652291 00004

[2]K Nagel,M Schreckenberg.A cellular automaton model for freewaytraffic[J].Journal de physique I,1992,2 ( 12 ) : 2221- 2229.

[3]Min HAN,Jinliang LIU. The congestion condition and solution of Tian Jin. Urban Roads Bridges \& Flood Control,2013(9):169-171. 